incidence and risk factors of oesophageal cancer by histological subtypes using data from 178 countries.

Methods The data on the incidence of oesophageal cancer by histological types in 2018 were estimated from GLOBALCAN and Cancer Incidence in Five Continents (CI5). Age-standardized rates (ASRs) for oesophageal cancer incidence by histological subtypes were evaluated by Segi-Doll population. The prevalence of tobacco use, alcohol drinking, physical inactivity, obesity, diabetes, and lipid disorders for each country were retrieved from the Global Health Observatory. The association between the ratio of histological subtypes and risk factors was examined by multivariable linear regression.

Results We estimated a total of 63,470 (12.6\%) and 502,669 new cases of oesophageal adenocarcinoma (AC) and squamous cell carcinoma (SCC) in 2018, respectively. The incidence among males was 3.6-fold and 2.2-fold of that among females for AC and SCC, respectively. The highest AC:SCC ratio was found in the UK (ratio 2.880, ASR 7.5), New Zealand (2.667, 4.2), the Netherlands (2.536, 7.7), Bahrain (2.143, $0.9)$, and Canada $(2.000,3.7)$ among males (figure 1). As for females, the highest AC:SCC ratio was observed in Moldova $(1.000,0.2)$, the Netherlands $(0.800,1.2)$, Iceland $(0.750$; $0.5)$, the UK $(0.700 ; 1.4)$, and Cyprus $(0.667 ; 0.3)$. A higher AC:SCC ratio was associated with a higher prevalence of obesity (male: $\beta 0.039$, 95\% CI 0.023 to 0.055 ; female: 0.009 , 0.004 to 0.146 ) and high cholesterol (male: 0.028, 0.010 to 0.047; female: $0.011,0.004$ to 0.019 ); but a lower prevalence of tobacco use (male: $-0.007,-0.014$ to -0.001 ) and diabetes (male: $0.009,0.004$ to 0.146 ; female: $-0.021,-0.038$ to 0.003).

Conclusions While SCC is the predominant subtype of oesophageal cancer, the incidence of AC has surpassed SCC in a substantial proportion of countries, probably due to the increasing prevalence of obesity and metabolic disorders. Future research should investigate the reasons behind these epidemiological changes.

\section{IDDF2020-ABS-0141 THE GUT MICROBIOME AND SERUM METABOLOME ORCHESTRATE HEALTHY AGING AND LONGEVITY WITH NOVEL IMPLICATIONS FOR RENAL FUNCTION}

\begin{abstract}
${ }^{1}$ Zhiming Li*, ${ }^{2}$ Liang Sun, ${ }^{3}$ Caiyou Hu, ${ }^{1}$ Jiahong Ding, ${ }^{2}$ Qi Zhou, ${ }^{1}$ Yuzhe Sun, ${ }^{1}$ Rui Li, ${ }^{1}$ Hefu Zhen, ${ }^{4}$ Shuqin Sun, ${ }^{4}$ Jianmin Zhang, ${ }^{1}$ Chuanfa Liu, ${ }^{1}$ Mingyan Fang, 'Zhihuan Chen, ${ }^{3}$ Yuan Lv, ${ }^{4}$ Qizhi Cao, ${ }^{4}$ Yanan Sun, ${ }^{5}$ Zezhi Huang, ${ }^{2}$ Ruiyue Yang, ${ }^{2}$ Jun Dong, ${ }^{5}$ Ranhui Gong, ${ }^{5} J u n c h u n$ Li, ${ }^{1}$ Jie Ruan, ${ }^{1}$ Haorong Lu, ${ }^{3}$ Guofang Pang, ${ }^{3}$ Benjin He, ${ }^{3}$ Ninghu Li, ${ }^{1} \mathrm{TaO} \mathrm{Li}$, ${ }^{1}$ Wenbin Xue, ${ }^{1}$ Yan Li, ${ }^{1}$ Juan Shen, ${ }^{2}$ Fan Yang, ${ }^{2}$ Cheng Zhao, ${ }^{3}$ Qinghua Liang, ${ }^{1}$ Mingrong Zhang, ${ }^{2}$ Chen Chen, ${ }^{2}$ Huan Gong, ${ }^{2}$ Huiping Yuan, ${ }^{1}$ Yong Hou, ${ }^{1}$ Jian Wang, ${ }^{2}$ Ying Zhang, ${ }^{1}$ Huanming Yang, 'Shida Zhu, ${ }^{1}$ Jianping Cai, ${ }^{1}$ Liang Xiao, ${ }^{6}$ Haiyun Guo, ${ }^{6}$ Peng Zhao, ${ }^{2}$ Jian Li, ${ }^{1}$ Susanne Brix Pedersen, ${ }^{1}$ Xun $\mathrm{Xu}$, ${ }^{1} \mathrm{Huijue}$ Jia, ${ }^{1}$ Karsten Kristiansen, ${ }^{2}$ Ze Yang. 'BGl, China; ${ }^{2}$ The MOH Key Laboratory of Geriatrics, Beijing Hospital, National Center of Gerontology, China; ${ }^{3}$ Jiangbin Hospital, China; ${ }^{4}$ School of Gerontology, Binzhou Medical University, China; ${ }^{5}$ Office of Longevity Cultural, People's Government of Yongfu County, China; ${ }^{6}$ Department of International Exchange and Cooperation, Kunming Medical University, China
\end{abstract}

\subsection{6/gutjnl-2020-IDDF.87}

Background Aging is closely associated with the occurrence and development of many complex diseases such as cardiovascular conditions, type 2 diabetes, gastrointestinal (GI) and renal diseases. Detecting changes as early as possible in the human body due to aging is thus crucial significant to potentially mitigate its impacts on physical health.
Methods Here, we present a comprehensive metagenome association study and serum metabolomics profiling in a registry Guangxi-Longevity cohort aged from 20-111 years $(n=151)$ and Kunming cohort ages ranging from 20-80 years $(n=80)$.

Results We identified uremic toxins as key factors in serum metabolomics highly associated with aging, and this finding has been validated in an independent Kunming-Aging cohort aged from $20-80$ years $(n=80)$. We also observed that agingassociated systemic inflammation levels were positively associated with uremic toxins. Moreover, the increased Escherichia coli, Odoribacter splanchnicus, Bilophila wadsworthia and Parabacteroides spp. abundances were related to serum levels of uremic toxins, and the accumulating rate of uremic toxins and specific microbial species was robustly much slower in centenarians than in nonagenarians. We further investigated the frailty and health status in long-living individuals aged above 90 years, and found that the frailty status might be a putative extreme aging phenomenon characterized by novel uremic toxin accumulation patterns.

Conclusions Our findings reveal novel potential links between gut microbiota alterations, uremic toxins and aging, and highlight the preponderance of gut microbiota and serum metabolism in aging.

\section{IDDF2020-ABS-0142 PERFORMANCE INDICATORS OF ORGANISED COLORECTAL CANCER SCREENING PROGRAMMES USING FAECAL IMMUNOCHEMICAL TESTS AND COLONOSCOPY: A SYSTEMATIC REVIEW AND META-ANALYSIS}

${ }^{1}$ Hanyue Ding ${ }^{*},{ }^{1}$ Jiaye Lin, ${ }^{1}$ Zijun Xu, ${ }^{1}$ Xiao Chen, ${ }^{2}$ Harry Haoxiang Wang, ${ }^{1}$ Liwen Huang, ${ }^{1}$ Junjie Huang, ${ }^{3}$ Zhijie Zheng, ${ }^{1}$ Martin Chi-sang Wong. ${ }^{1} J C$ School of Public Health and Primary Care, Faculty of Medicine, Chinese University of Hong Kong, China; ${ }^{2}$ School of Public Health, Sun Yat-Sen University, China; ${ }^{3}$ School of Public Health, Peking University, China

\subsection{6/gutjnl-2020-IDDF.88}

Background The success of population-based colorectal cancer (CRC) screening is dependent on the optimal achievement of various targets. We estimated the pooled performance indicators of CRC screening programmes that used faecal immunochemical tests (FIT) as a primary screening modality and colonoscopy as a subsequent confirmatory test in various countries.

Methods We searched PubMed, Ovid MEDLINE, Embase, and Cochrane from inception to 1st Jan 2020. We included original articles published in the English language describing population-based CRC screening programme that used FIT and colonoscopy, and relevant national CRC screening reports by hand searching. We extracted data to pool early performance indicators, including participation rate, invalid FIT rate, FIT positive rate, adenoma/CRC detection rate of FIT, colonoscopy compliance rate, rate of adequate bowel preparation, colonoscopy completion rate, colonoscopy complication rate, and positive predictive values (PPV) of FIT for adenoma/CRC. We used Metaprop to conduct a meta-analysis via $\mathrm{R}$ software (version 3.6.3). The Freeman-Tukey double arcsine transformation was used to stabilise the variances, and a random-effects model was used to pool the rates with proportions.

Results A total of 85 studies (64 articles and 21 reports) were included in this meta-analysis. The pooled participation rate was 52.2\%, and the pooled proportion of invalid FIT was 
1.0\%. The FIT positive rate was $7.0 \%$, the adenoma detection rate was $21.8 \%$, and the CRC detection rate was $2.5 \%$. In addition, the colonoscopy compliance rate $(79.1 \%)$, rate of adequate bowel preparation (86.3\%), and the colonoscopy completion rate $(93.1 \%)$ were high. We found the pooled complication rate of $0.3 \%$, with PPVs of FIT being $39.2 \%$ (for adenoma) and $4.7 \%$ (for CRC). The range of all indicators varied substantially. In subgroup analysis, a higher FIT cut-off value indicated lower positivity and lower adenoma detection rate. Compared with CRC screening programmes with one FIT, those with two FIT had lower participation rate $(48.8 \%$ vs $55.5 \%)$ but higher positive rate $(8.9 \%$ vs $5.8 \%)$ and adenoma detection rate $(21.8 \%$ vs $17.3 \%$ ).

Conclusions Our findings reported the pooled performance indicators of different CRC screening programmes. The summary measures could inform the benchmarking of performance indicator targets across different CRC screening programmes.

\section{IDDF2020-ABS-0143 A SINGLE-CENTRE PROSPECTIVE AUDIT OF INPATIENT CARE FOR ADULTS WITH ULCERATIVE COLITIS}

${ }^{1}$ Wilson Siu*, ${ }^{1}$ Hannah Lambert, ${ }^{2}$ Jason On, ${ }^{1}$ Malcolm Smith. ${ }^{1}$ Department of Digestive Disorders, Aberdeen Royal Infirmary, UK; ${ }^{2}$ Department of Colorectal Surgery, Aberdeen Royal Infirmary, UK

\subsection{6/gutjnl-2020-IDDF.89}

Background Acute severe ulcerative colitis (UC) is a potentially life-threatening presentation and requires coordinated multidisciplinary management. The aim of our prospective audit was to evaluate the inpatient management and outcome of patients with UC.

Methods All consecutive patients admitted to Aberdeen Royal Infirmary for treatment of acute UC were prospectively recruited in the study over a 6 months period. All patients were followed up for a minimum of 3 months from the discharge date. Patient's demographics, clinical data, endoscopic assessment, medical and surgical treatment details were collected. The clinically significant outcome was defined as steroid treatment failure requiring surgery or rescue therapies (ciclosporin or infliximab) despite intravenous steroid therapy. Statistical comparisons were made using Non-parametric Mann-Whitney test and Fishers Exact test.

Results 27 patients (15 females; median age 41 years (IQR 31-63)) were admitted for treatment of UC during the 6 months period. 23 patients had severe UC as per Truelove and Witts Score. 19 patients had pre-existing diagnosis of UC prior admission. Faecal calprotectin was checked in 3 patients and were all $>1000 \mathrm{ug} / \mathrm{g}$.

All patients received a minimum of 3 days of intravenous steroid. 10 patients were non-responsive to steroid therapy. 3 patients received rescue infliximab while 1 patient received ciclosporin. 7/27 (26\%) required in-patient colectomy during the study period. Previous diagnosis of UC, previous admission or mesalazine use were not associated with steroid treatment failure. Only bloody stool frequency at Day 3 had a statistically significant association $(p=0.03)$. The median (IQR) bloody stool frequency was $5(1.8-10.3)$ for those who were in the steroid failure group compared to $3(0-3)$ for those in the steroid responder group.

Conclusions In our prospective study, the in-hospital colectomy rate was $7 / 27(26 \%)$ for acute presentation of UC. Systemic oral steroid use prior to admission was associated with steroid treatment failure but was not statistically significant $(p=0.05)$. Future study with a larger sample size could perhaps identify more clinical and laboratory variables that could be useful to stratify patients at risk of steroid failure.

\section{IDDF2020-ABS-0144 CATASTROPHIC EMERGENCY EVENTS OF COMPLICATED ULCERATIVE COLITIS: AN IMPACT ON CLINICAL OUTCOME}

${ }^{1}$ Ida Ayu Setyawati Sri Krishna Dewi ${ }^{*},{ }^{2}$ Budhi Ida Bagus. 'Medical Faculty, Pendidikan Ganesha University, Indonesia; ${ }^{2}$ Department of Surgery, Sebelas Maret University, Indonesia

\subsection{6/gutjnl-2020-IDDF.90}

Background As one of the inflammatory bowel diseases case, ulcerative colitis (UC) has many clinical manifestations, including usual clinical symptoms or emergency presentation of this part of IBD. Surgery is the last treatment option of ulcerative colitis, in the era of better medication over the last decades, we could decrease the need for elective or emergency surgeries. The two most common emergency case was uncontrolled hemorrhage and colonic perforation.

Methods We would evaluate the emergency presentation of ulcerative colitis in our institution during the last 6 months from January until June 2020. All patients with already known as ulcerative colitis will be included in this study, clinical presentation and the surgical option will be recorded. The clinical outcome will be evaluated from inpatients mortality and postoperative morbidity if present.

Results During the last 6 months, we reported 1 case of 74 years old male with diffuse peritonitis due to hollow viscous perforation, free intraperitoneal air was found on abdominal $\mathrm{x}$-rays, the patient only has diarrhea symptom before and no history of specific medication for his ulcerative colitis. This case was found incidentally on an emergency setting, and he had sepsis condition with cardiopulmonary comorbidity. Surgery has been done, and total colectomy was the option on this case with stoma creation. Cardiopulmonary support already has been given in the intensive care unit and no recovery from the prolonged sepsis after the first 24 hours. This complicated UC condition with late presentation case has an unfavorable outcome on catastrophic emergency condition.

Conclusions Ulcerative colitis has a life-threatening emergency presentation, late presentation, and preoperative comorbidities play an important role in the unfavorable outcome on complicated ulcerative colitis cases.

\section{IDDF2020-ABS-0145 COMBINATION OF ANTIBIOTICS AS ADJUVANT THERAPY IN ACUTE SEVERE ULCERATIVE COLITIS: A RANDOMIZED TRIAL}

Shubhra Mishra*, Harshal Mandavdhare, Harjeet Singh, Arup Choudhury, Jimil Shah, Sant Ram, Dimple Kalsi, Jayanta Samanta, Kaushal Prasad, Arun Sharma, Usha Dutta, Vishal Sharma. Postgraduate Institute of Medical Education and Research, India

\subsection{6/gutjnl-2020-IDDF.91}

Background Some studies have suggested that targeted combination therapy with antibiotics could improve response in active ulcerative colitis, but similar data is not available in acute severe UC (ASUC). 